\title{
Premières observations de UVSQ-SAT
}

UVSQ-SAT est un nanosatellite français au standard CubeSat (Twiggs, 2000), un format de satellite défini en 1999 par l'université d'État polytechnique de Californie et l'université de Stanford (États-Unis). UVSQ-SAT est un démonstrateur pédagogique, technologique et scientifique dédié à l'observation de la Terre et du Soleil. Il a été imaginé, conçu, réalisé et testé par le Laboratoire atmosphères, milieux, observations spatiales (Latmos), en collaboration avec ses partenaires académiques et industriels, et la communauté radioamateur francophone. De la taille d'un cube de $10 \mathrm{~cm}$ d'arête et
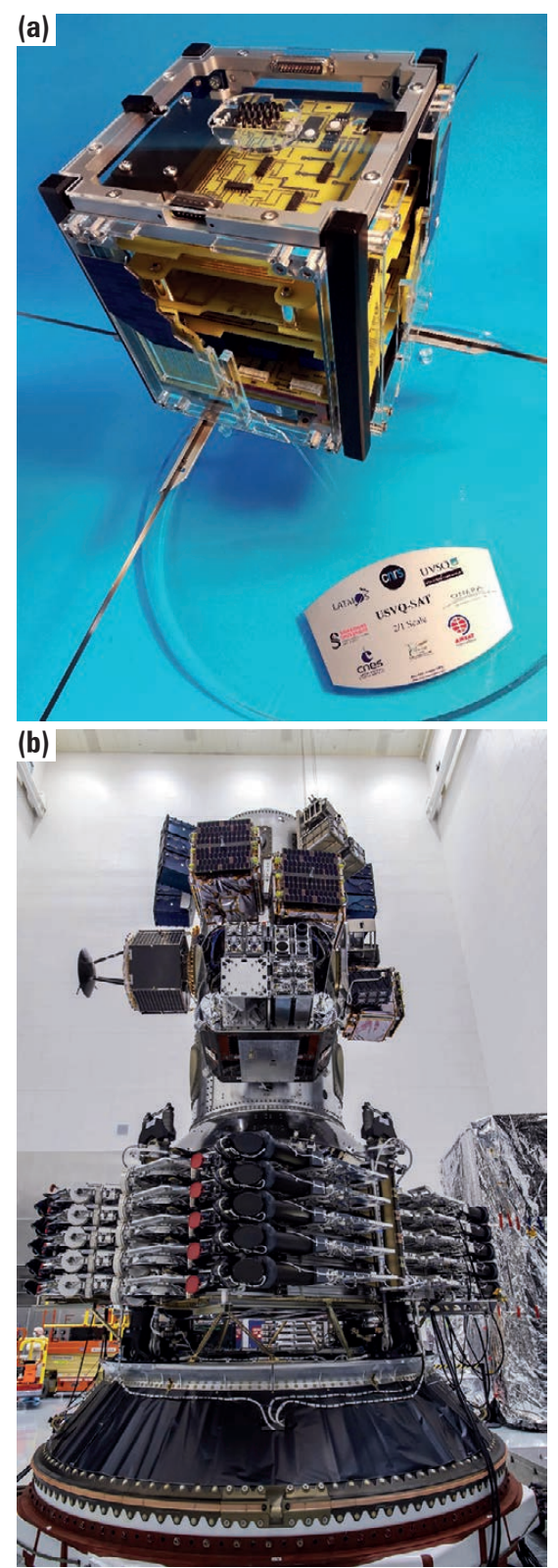

Figure 1. (a) Maquette représentative du satellite UVSO-SAT. (b) Mission Transporter 1 avec à son bord 143 satellites commerciaux et gouvernementaux, dont UVSO-SAT. pesant à peine $2 \mathrm{~kg}$, ce satellite a été mis en orbite le 24 janvier 2021 par le lanceur Falcon 9 (SpaceX) avec la mission Transporter 1 (figure 1). Depuis, le satellite est en orbite héliosynchrone autour de la Terre à une altitude d'environ $540 \mathrm{~km}$ pour une durée de vie opérationnelle souhaitée d'au moins un an.

\section{Objectifs de la mission}

Un des objectifs de la mission est de mettre en œuvre un démonstrateur agile permettant de réaliser des mesures du bilan radiatif de la Terre au sommet de l'atmosphère (Meftah et al., 2020). Cette question scientifique est un problème extrêmement complexe. Les premiers bilans relativement détaillés des échanges énergétiques de notre planète et de son atmosphère ont été publiés par Kiehl et Trenberth (1997). Une mise à jour du bilan radiatif de la Terre a été réalisée par Stephens et al. (2012). Celui-ci n'est pas à l'équilibre $\mathrm{du}$ fait du réchauffement climatique en cours. Le déséquilibre énergétique de la Terre correspond à la différence entre le rayonnement solaire incident et le rayonnement solaire réfléchi par la Terre associé au rayonnement sortant à grande longueur d'onde qu'elle émet thermiquement. Une étude publiée récemment (von Schuckmann et al., 2020) estime le déséquilibre énergétique de la Terre à $+0,87 \pm$ $0,12 \mathrm{Wm}^{-2}$ au cours de la période 20102018 , contre $+0,47 \pm 0,10 \mathrm{Wm}^{-2}$ au cours de la période 1971-2018.

Le déséquilibre énergétique de la Terre est un paramètre clé pour caractériser le réchauffement climatique qui est complémentaire des autres paramètres couramment utilisés (concentration de $\mathrm{CO}_{2}$ et température à la surface de la Terre). Dès lors, mesurer ce déséquilibre représente un impératif pour la surveillance du changement climatique, auquel les missions spatiales, comme UVSQ-SAT du projet de constellation de petits satellites (Meftah et al., 2020) dénommée Terra-F, essayent de répondre. Il est plus que nécessaire de poursuivre nos efforts pour mettre en œuvre de nouveaux systèmes spatiaux d'observation, car le déséquilibre énergétique de la Terre représente un " réservoir 》 de réchauffement climatique qui se manifestera même si le forçage radiatif venait à être stabilisé. Les mesures directes des variations du déséquilibre énergétique de la Terre sont d'une importance primordiale pour déterminer le rythme du changement climatique aux échelles régionale et mondiale.

\section{Premières observations}

Depuis février 2021, le satellite UVSQ$S A T$ mesure entre autres le rayonnement solaire réfléchi par la Terre et le rayonnement sortant à grande longueur d'onde. UVSQ-SAT ne dispose pas de système actif de contrôle d'attitude. Dès lors, il est équipé de deux capteurs de mesure de flux sur chacune de ses faces afin de réaliser les mesures en permanence, soit douze capteurs au total. Ceux-ci sont fondés sur un principe qui consiste à réaliser des discontinuités thermiques structurelles sur une thermopile planaire. Sur chaque face du satellite, une thermopile à base de nanotubes de carbone observe la Terre et le Soleil. Les nanotubes de carbone déposés sur ces thermopiles permettent d'absorber tous les rayonnements incidents qu'ils soient de courtes ou grandes longueurs d'onde (avec une absorption très proche de 1). La réflectivité bidirectionnelle du revêtement en nanotubes de carbone a été mesurée avant le lancement avec un goniophotomètre. Les propriétés de ce revêtement optique présentent une faible dépendance angulaire. Les rayonnements absorbés génèrent alors des gradients de température sur la thermopile qui délivre ainsi une tension proportionnelle au flux. En période d'éclipses, ces détecteurs mesurent uniquement le rayonnement sortant de la Terre à grande longueur d'onde. Hors éclipses, les thermopiles mesurent l'éclairement solaire incident, le rayonnement solaire réfléchi par la Terre et le rayonnement sortant à grande longueur d'onde. Sur chaque face du satellite a aussi été installée une thermopile à base de réflecteurs solaires optiques qui permet de mesurer essentiellement le rayonnement sortant à grande longueur d'onde. L'ensemble des douze thermopiles représentent des détecteurs à champ de vue large. Ils réalisent des mesures dans un hémisphère complet.

La méthode de Gristey et al. (2017), fondée sur les harmoniques sphériques, sera utilisée pour reconstruire plus finement les cartes d'albédo et de rayonnement sortant à grande 
longueur d'onde. La résolution spatiale attendue pourrait être de l'ordre de $1000 \mathrm{~km}$ avec une incertitude de quelques centaines de milliwatts par mètre carré pour une durée suffisante de mesures. Aujourd'hui, la reconstruction de chaque cartographie (albédo et rayonnement sortant à grande longueur d'onde) repose sur une fonction gaussienne dont les paramètres sont définis par l'altitude du satellite et son champ de vue. Par ailleurs, une méthode fondée sur un algorithme d'apprentissage profond (deep learning) a été développée pour obtenir les variations d'attitude du satellite afin de mieux déterminer l'albédo de la Terre et le rayonnement sortant à grande longueur d'onde. L'attitude du satellite peut aussi être reconstruite à partir des mesures d'une centrale inertielle. UVSQ-SAT étant équipé d'un gyromètre pour la mesure de la vitesse angulaire, d'un accéléromètre pour la mesure de la gravité et de l'accélération linéaire, ainsi que d'un magnétomètre pour mesurer le champ magnétique terrestre. Le magnétomètre permet de mesurer la direction et l'intensité du champ magnétique. L'objectif est de fusionner ces données inertielles et magnétiques afin de reconstruire au mieux l'attitude du satellite. La figure 2 montre l'intensité du champ magnétique de la Terre mesuré par UVSQ-SAT grâce à ses différents capteurs.

Des résultats préliminaires pour le rayonnement solaire réfléchi par la Terre et le rayonnement sortant à grande longueur d'onde ont été obtenus en appliquant les méthodes décrites dans Meftah et al. (2020). La figure 3 montre une cartographie de l'albédo de la Terre (février 2021) obtenue à partir des instruments de UVSQ$S A T$. L'albédo est dû à la réflexion $\mathrm{du}$ rayonnement solaire incident par les surfaces et par l'atmosphère. La contribution de l'atmosphère est due aux molécules (diffusion Rayleigh qui est plus forte dans les longueurs d'ondes courtes) et aux nuages, et correspond environ aux deux tiers de l'albédo total. Le rayonnement solaire total au sommet de l'atmosphère est plus fort dans les tropiques. L'albédo en revanche est plus élevé aux latitudes polaires à cause de l'inclinaison du rayonnement incident mais aussi de la présence de neige et de glace au sol. Il y aussi des différences locales dues à l'albédo des régions nuageuses (zone intertropicale de convergence) ou du sol (Sahara). L'albédo du sol dépend fortement de sa nature, il peut être très élevé pour de la neige fraîche (environ



Figure 2. Intensité du champ magnétique de la Terre mesurée par UVSO-SAT pour le mois de février 2021. Les étalonnages sont en cours de validation pour consolider les biais de mesures absolus.

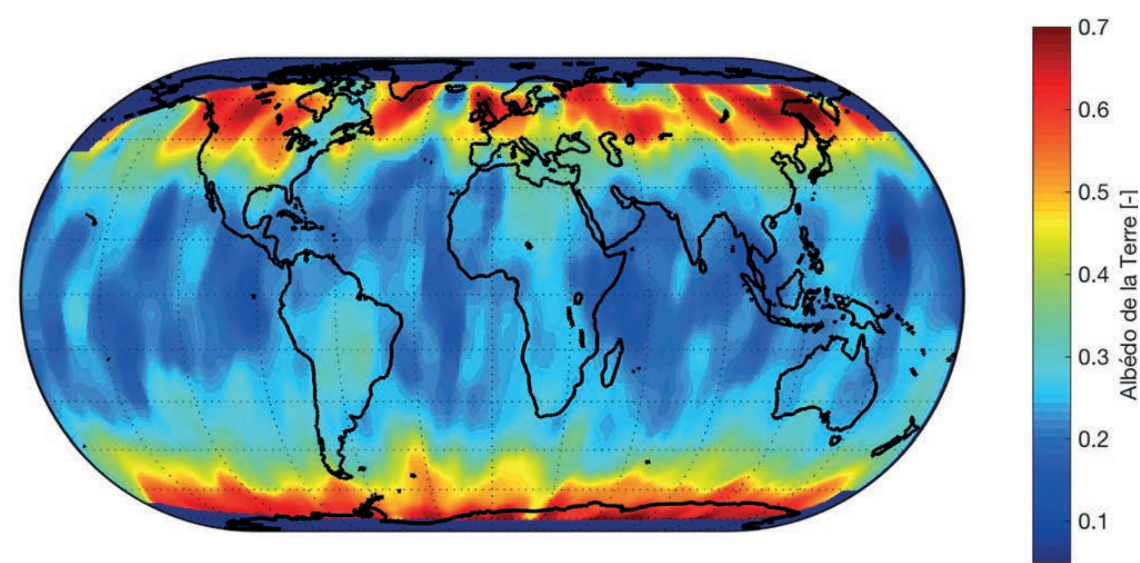

Figure 3. Albédo de la Terre mesuré par UVSQ-SAT pour le mois de février 2021. Les zones en bleu foncé au niveau des pôles correspondent à des régions sans données.

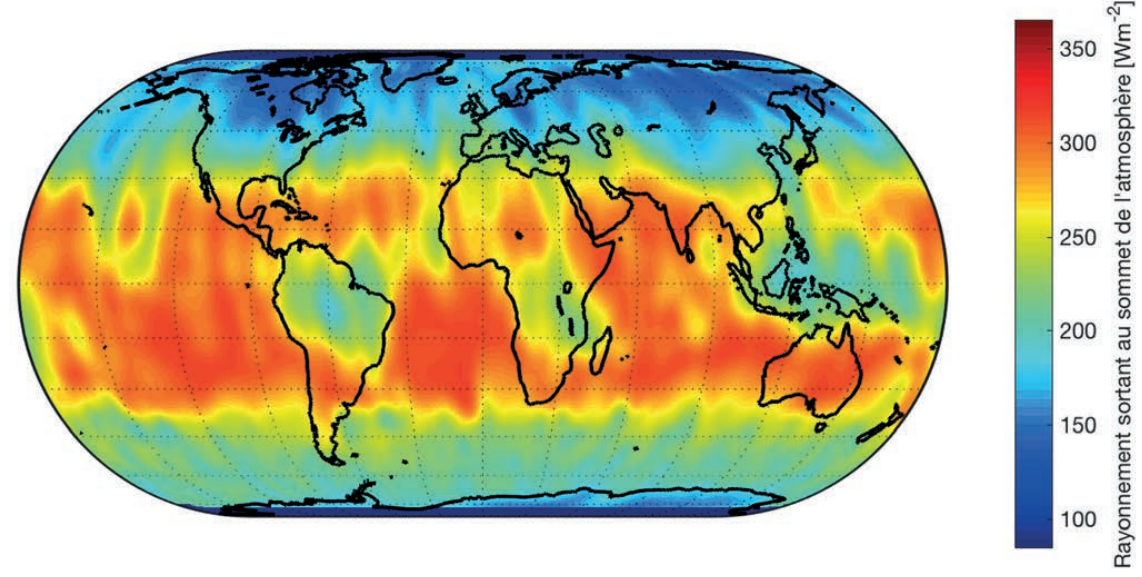

Figure 4. Rayonnement sortant au sommet de l'atmosphère de la Terre à grandes longueurs d'onde pour le mois de février 2021

0,75 ) et faible pour de la végétation (inférieur à 0,2$)$. L'albédo de l'océan, qui dépend de la distribution des vagues, reste faible, de l'ordre de 0,1 . Compte tenu de toutes ces dépendances, il est intéressant de suivre l'évolution de l'albédo de la planète au cours du temps.

Le rayonnement sortant au sommet de l'atmosphère de la Terre à grande longueur d'onde (figure 4) a lui aussi une dépendance en latitude. Les hautes latitudes de la Terre, qui sont plus froides, émettent moins de rayonnement infrarouge. Les régions humides tropicales sont nettement visibles. Dans les régions tropicales et équatoriales, le faible rayonnement sortant est dû à la présence de nuages à haute altitude. Ceux-ci absorbent le rayonnement émis par la surface de la Terre. Dès lors, comme ils sont froids, ils émettent vers l'espace un faible rayonnement sortant. 
Les premiers résultats de la mission sont très encourageants et montrent clairement que les petits satellites représentent une méthode rapide pour répondre à des questions scientifiques clés. À l'ère du NewSpace, c'est-à-dire une ère bénéficiant des progrès technologiques de la miniaturisation et de coûts raisonnables d'accès à l'espace, cette mission montre clairement qu'une constellation de petits satellites dédiés à la mesure du déséquilibre énergétique de la Terre est envisageable.

Malgré l'urgence climatique, il reste difficile d'obtenir des financements pour les missions satellitaires. Les nanosatellites offrent donc une opportunité à moindre coût pour la mise en œuvre de constellations. Ces missions doivent s'appuyer sur la mise en œuvre de constellations de satellites afin d'assurer une couverture spatiale et temporelle suffisante. Les constellations de satellites dédiées à l'étude du climat et à la science représentent aujourd'hui une évidence (Baker et al., 2020). Le prototype UVSQ-SAT ouvre la voie à une potentielle constellation Terra- $F$ composée de 50 petits satellites ayant les performances nécessaires pour répondre au besoin scientifique.

D'autres solutions pour mesurer le déséquilibre énergétique de la Terre sont possibles en utilisant deux satellites traditionnels placés aux points de Lagrange L1 et L2 afin d'effectuer des mesures des portions éclairées et non éclairées de notre planète. La principale ambition est de réaliser des mesures du déséquilibre énergétique de la Terre au sommet de l'atmosphère et de sa variabilité dans le temps afin de détecter toute tendance globale à long terme avec une stabilité d'au moins $\pm 0,2 \mathrm{~W} \mathrm{~m}^{-2}$ par décennie, mais aussi de pouvoir obtenir des résolutions spatiales de quelques dizaines de kilomètres et temporelles de trois heures avec une précision de quelques watts par mètre carré.

UVSQ-SAT et sa potentielle future constellation abordent la même thématique scientifique que le programme Forum (Far-infrared Outgoing Radiation Understanding and Monitoring) du programme Earth Explorer de l'Esa, mais sous un angle complètement différent.

Mustapha Meftah, Philippe Keckhut Latmos, CNRS / UVSQ / Université Paris-Saclay/Sorbonne Université

\footnotetext{
Pour en savoir plus

http://uvsq-sat.projet.latmos.ipsl.fr

Baker D., Chandran A., Chang L., Macdonald M., Meftah M., Millan R., Park J. H., Kumar P., Price C., von Steiger R., Wu J. et al., 2020. An international constellation of small spacecraft. Space Research Today, 208, 23-28.

Gristey J.J., Chiu J.C., Gurney R.J., Han S.C., Morcrette C.J., 2017. Determination of global Earth outgoing radiation at high temporal resolution using a theoretical constellation of satellites. J. Geophys. Res., 122, 1114-1131.

Kiehl J.T., Trenberth K.E., 1997. Earth's annual global mean energy budget. Bull. Am. Meteorol. Soc., 78, $197-197$.

Meftah M., Damé L., Keckhut P., Bekki S. et al., 2020. UVSQ-SAT, a pathfinder CubeSat mission for observing essential climate variables. Remote Sens., 12, 92. doi: 10.3390/rs12010092

Stephens G.L., Li J., Wild M., Clayson C.A., Loeb N., Kato S., L'Ecuyer T., Stackhouse P.W., Lebsock M., Andrews T., 2012. An update on Earth's energy balance in light of the latest global observations. Nat. Geosci., 5, 691-696.

Twiggs R.J., 2000. Space system developments at Stanford University: From launch experience of microsatellites to the proposed future use of picosatellites. SPIE 4136. Small Payloads in Space, 4136, 79-86. doi: 10.1117/12.406646

von Schuckmann K., Cheng L., Palmer M.D. et al., 2020. Heat stored in the Earth system: Where does the energy go? Earth Syst. Sci. Data, $12,2013-2041$.
}

\section{Arktika-M1, un satellite météorologique russe en orbite excentrée}

Les orbites des satellites météorologiques ont été largement évoquées dans cette revue (Capderou, 2017a, b). L'actualité - le lancement du satellite météorologique russe Arktika-M1, le premier du programme Arktika-M - nous incite à revenir sur le sujet, car l'orbite de cette famille de satellites est très originale pour ce type de mission.

Les (magnifiques) images de Météosat ont vulgarisé, pratiquement banalisé, le concept de satellite géostationnaire. Tout honnête homme sait à présent que l'orbite de ces satellites est équatoriale et circulaire. Leur altitude est très élevée : 5,61 rayons terrestres, soit environ $36000 \mathrm{~km}$. Et on constate vite, en observant les images (un disque représentant presque la moitié du globe terrestre) que les régions près des pôles sont absentes ou très mal représentées.

Tableau 1. Caractéristiques orbitales pour deux types de satellites (voir Capderou, 2017a) : a le demigrand axe, e l'excentricité (sans dimension), $i$ l'inclinaison, $h_{a}$ l'altitude à l'apogée et $h_{p}$ l'altitude au périgée.

\begin{tabular}{lccccc} 
& $\boldsymbol{a}(\mathbf{k m})$ & $\boldsymbol{e}$ & $\boldsymbol{i}\left({ }^{\circ}\right)$ & $\boldsymbol{h}_{\mathrm{a}}(\mathbf{k m})$ & $\boldsymbol{h}_{\mathrm{p}}(\mathbf{k m})$ \\
Molnya classique & 26552,8 & 0,75 & 63,4 & 40089 & 260 \\
\hline Arktika-M1 & 26552,8 & 0,72 & 63,3 & 39310 & 1073 \\
\hline
\end{tabular}

On admet qu'au-delà de $55^{\circ}$ de latitude nord les images ne sont pas correctement exploitables. En ce qui concerne l'Europe, un satellite géostationnaire ne présente pas d'intérêt pour l'Islande, l'Écosse, les pays scandinaves et la Russie.

Ce fait, les Russes l'ont intégré depuis longtemps, dès le début de l'ère spatiale, qui avait si brillamment commencé pour l'URSS. Pour contourner ces problèmes de position géographique intangible, les ingénieurs soviétiques ont inventé l'orbite Molnya. Cette orbite est une ellipse très excentrée dont les caractéristiques sont notées dans le tableau 1. La période est proche de 12 heures; plus exactement, elle est égale à un demi-jour sidéral, soit 717,72 minutes. Une autre propriété de cette orbite est qu'elle est " phasée » (Capderou 2012, 2014), c'est-à-dire que sa trace repasse perpétuellement sur elle-même, jour après jour.

L'application de la deuxième loi de Kepler montre que le satellite se déplace très lentement près de l'apogée et très rapidement près du périgée ; 